\title{
WEIGHTED ESTIMATES FOR FRACTIONAL POWERS OF PARTIAL DIFFERENTIAL OPERATORS
}

BY

\author{
RAYMOND JOHNSON
}

\begin{abstract}
It is shown that fractional powers defined by the wave polynomial $P(\xi)=\xi_{1}^{2}+\cdots+\xi_{p}^{2}-\xi_{p+1}^{2}-\cdots-\xi_{n}^{2}$, defined in terms of Fourier transforms by $\widehat{T^{\lambda} f}=|P(\xi)|^{\lambda} \hat{f}$, are in the Bernstein subalgebra of functions with integrable Fourier transforms for $\lambda>(n-1) / 2$, provided $f \in C_{c}^{m}$ with $m$ large enough. The proof uses embedding theorems for Besov spaces and Stein's theorem on interpolation of analytic families of operators.
\end{abstract}

In recent papers [3], [4] studying the Plancherel formula for parabolic subgroups of real rank one simple Lie groups, Keene, Lipsman and Wolf have needed mapping properties of the operator defined by

$$
T^{\lambda} f(x)=(2 \pi)^{-n} \int e^{i\langle x, \xi\rangle}|P(\xi)|^{\lambda} \hat{f}(\xi) d \xi .
$$

They were particularly interested in the question of when $T^{\lambda}$ maps the space of $l$-times continuously differentiable functions with compact support into the space of integrable functions. When $P$ is the elliptic polynomial $P(\xi)=\xi_{1}^{2}+\cdots+\xi_{n}^{2}$, they proved that $T^{\lambda}$ has the desired property for any $\lambda>0$. We first give a sharper version of this result by showing that $|\xi|^{2 \lambda} \hat{f}$ actually belongs to the Bernstein subalgebra of the Fourier algebra of functions with integrable Fourier transforms and indicate the relations between these results and weighted estimates for the Riesz potential. We then use a weighted estimate for the Fourier transform to prove an embedding theorem for a homogeneous isotropic unweighted Besov space with a weighted $L^{2}$ space. We combine this with Stein's theorem on interpolation of analytic families of operators to prove that if $\lambda>n / 2-1 / 2,|P(\xi)|{ }^{\lambda} \hat{f}$ is in the Bernstein algebra, where $P(\xi)=\xi_{1}^{2}+\cdots+\xi_{p}^{2}-\xi_{p+1}^{2}-\cdots-\xi_{n}^{2}$. This result is not quite the result conjectured (i.e., that this should be true for $\lambda>n / 2-1$ ), but it does capture the case $\lambda=n / 2$ needed in [4].

The weighted estimates used could be replaced by simple direct estimates. During the course of preparing this manuscript, the author has benefited from conversations with L. Carleson, C. Herz and N. Lohoué.

0. Notation. Our notation will be standard. We will mainly work on $L^{p}$ spaces on $R^{n}$ where

$$
\begin{array}{rlrl}
L^{p}\left(R^{n}\right) & =\left\{f \text { measurable }\left.\left|\int\right| f\right|^{p} d x<+\infty\right\}, & & 1 \leqslant p<\infty, \\
& =\{f \text { measurable |ess } \sup |f|<+\infty\}, & p=\infty
\end{array}
$$

Received by the editors November 14, 1979.

1980 Mathematics Subject Classification. Primary 46F12; Secondary 42B99, 46E35. 
Our convention for the Fourier transform is

$$
\hat{f}(\xi)=\int \exp (-i\langle x, \xi\rangle) f(x) d x, \quad f \in L^{1}\left(R^{n}\right) .
$$

The homogeneous Besov spaces are closely connected with the Riesz potentials. Let $\tau_{h}$ denote the translation operator and $\Delta_{h}$ the first difference operator, defined respectively by

$$
\tau_{h} f(x)=f(x-h), \quad \Delta_{h} f(x)=\left(\tau_{-h}-I\right) f .
$$

If $0<\alpha<1,1 \leqslant p, q \leqslant \infty, f \in \dot{B}_{p q}^{\alpha}$ if $\int\left(\left\|\Delta_{h} f\right\|_{p} /|h|^{\alpha}\right)^{q}|h|^{-n} d h<+\infty$ (with the obvious interpretation if $p$ or $q=\infty$ ). For an arbitrary real number $\alpha$, the homogeneous Besov spaces are defined by the Riesz potentials. The Riesz potential of order $\beta$ is the closure of the operator defined on a dense subset of $\mathcal{S}^{\prime}$, the dual space of the Schwartz space of rapidly decreasing functions, by

$$
\widehat{R^{\beta} f}(\xi)=|\xi|^{-\beta} \hat{f}(\xi)
$$

and the definition of $\dot{B}_{p q}^{\alpha}$ for $\alpha$ arbitrary is accomplished by $R^{\beta} \dot{B}_{p q}^{\alpha}=\dot{B}_{p q}^{\alpha+\beta}$.

The Fourier transform images of the Besov spaces are related to the spaces $K_{p q}^{a}$, where

$$
K_{p q}^{0}=\left\{f \text { measurable } \mid \sum_{-\infty}^{\infty}\left(\int_{2^{k}<|x|<2^{k+1}}|f(x)|^{p} d x\right)^{q / p}<+\infty\right\},
$$

and $f \in K_{p q}^{\alpha}$ if and only if $f(x)|x|^{\alpha} \in K_{p q}^{0}$.

For an arbitrary space $X$, we denote $\mathscr{F} X=\{\hat{f} \mid f \in X\}$, and another notation for $\mathscr{F} L^{1}$ is $A$,

$$
A\left(R^{n}\right)=\mathscr{F} L^{1}\left(R^{n}\right)=\left\{\hat{f} \mid f \in L^{1}\left(R^{n}\right)\right\} .
$$

We also use the Lorentz spaces, $L^{p, q}\left(R^{n}\right)$, defined as the set of measurable $f$ for which

$$
\int_{0}^{\infty}\left(t^{1 / p} f^{*}\right)^{q} \frac{d t}{t}<+\infty
$$

where $f^{*}$ is the decreasing rearrangement of $f$, the function with the property that

$$
\left|\left\{t \mid f^{*}(t)>\lambda\right\}\right|=|\{x|| f(x) \mid>\lambda\}|,
$$

where the measure on the left-hand side is Lebesgue measure on $(0, \infty)$ and that on the right-hand side is Lebesgue measure on $R^{n}$.

The operators that we shall consider are

$$
T^{\lambda} f(x)=(2 \pi)^{-n} \int \exp (i\langle x, \xi\rangle)|P(\xi)|^{\lambda} \hat{f}(\xi) d \xi, \quad f \in \delta\left(R^{n}\right),
$$

which are conventionally denoted by $\Delta^{\lambda}$ when $P(\xi)=\xi_{1}^{2}+\cdots+\xi_{n}^{2}$ since for $\lambda=k, T^{k}=\Delta^{k}$ is the $k$ th iterate of the Laplacian. Recall also that for $-n / 2<\lambda$ $<0, \Delta^{\lambda}$ coincides with the Riesz potential operator discussed above, $\Delta^{\lambda}=R^{-2 \lambda}$, and can also be defined by the convolution operator

$$
\Delta^{\lambda} f(x)=c_{\lambda} \int\left(f(y) /|x-y|^{n+2 \lambda}\right) d y, \quad f \in C_{c}^{\infty}\left(R^{n}\right) .
$$


1. Preliminaries. We recall the main results from [2] that we will be using. It is our intention to prove that $|\xi|^{\lambda} \hat{f} \in \dot{B}_{21}^{n / 2}$, the Bernstein subalgebra of the algebra of functions whose Fourier transforms are integrable. For a Besov space with positive $\alpha$, by Proposition 5.4 of [2], we know that

$$
\dot{B}_{22}^{\alpha} \cap L^{2} \subseteq \dot{B}_{21}^{n / 2}
$$

if $\alpha>n / 2$. Since it is routine to decide for which $\lambda,|\xi|^{\lambda} \hat{f} \in L^{2}$, we will concentrate on showing that it belongs to $\dot{B}_{22}^{\alpha}$. We will further use the trace theorems for Besov spaces to show that it suffices to prove the result for a sequence of dimensions $W_{j} \rightarrow \infty$ as $j \rightarrow \infty$. The trace theorem used is

TheOREM. If $s>1 / 2$, the map $f \mapsto f\left(x^{\prime}, 0\right), f \in C_{0}^{\infty}\left(R^{n}\right)$ extends to a bounded linear map of $\dot{B}_{22}^{s}\left(R^{n}\right) \rightarrow \dot{B}_{22}^{s-1 / 2}\left(R^{n-1}\right)$.

We have denoted for $x=\left(x_{1}, \ldots, x_{n}\right) \in R^{n}, x^{\prime}=\left(x_{1}, \ldots, x_{n-1}\right)$. Finally, it is convenient to use the weighted estimates of Muckenhoupt-Wheeden, especially the following.

TheOREM. Suppose $0<\gamma<n, 1<p<n / \gamma, 1 / q=1 / p-\gamma / n$. Then

$$
\left\|\left(R^{\gamma} f\right) V(x)\right\|_{q} \leqslant C\|f V\|_{p}
$$

$\Leftrightarrow V$ satisfies $A_{p q}$

$$
\sup _{Q}\left(\frac{1}{|Q|} \int_{Q} V(x)^{q} d x\right)^{1 / q}\left(\frac{1}{|Q|} \int_{Q} V(x)^{-p^{\prime}} d x\right)^{1 / p^{\prime}} \leqslant K,
$$

where the sup is taken over all cubes with sides parallel to the coordinate axes.

2. Results for $|\xi|^{\lambda} \hat{f}(\xi)$. Our main result for the elliptic case is given in Theorem 2.1.

THEOREM 2.1. If $\lambda+n / 2>0$, then $|\xi|^{\lambda} \hat{f} \in \dot{B}_{22}^{\gamma}$ for any $\gamma$ with $0<\gamma<\lambda+n / 2$, as long as $f \in C_{c}^{m}$, with $m \geqslant 2[\lambda / 2]+2$.

For negative $\lambda$, this is a restatement of the Muckenhoupt-Wheeden result cited in $\S 1$.

Proposition 2.2. If $0>a>-n / 2, f \in L_{|x| p r}^{p}, 1 / 2=1 / p+a / n$, then $|\xi| \hat{f} \hat{f} \in$ $\dot{B}_{22}^{\gamma},-n / 2<\gamma<a+n / 2$.

Proof. Recall that the $\dot{B}_{22}^{\gamma}$ norm of a function is equivalent to the $K_{22}^{\gamma}$ norm of its Fourier transform. Let $-b=a$, so that $0<b<n / 2$ and then note that

$$
\widehat{R^{b f}}=|\xi|^{-b} \hat{f}=|\xi|^{a} \hat{f}
$$

implying that the norm we wish to estimate is a weighted $L^{2}$ norm of $R^{b}$,

$$
\dot{B}_{22}^{\gamma}\left(|\xi|^{a} \hat{f}\right)=\left(\int\left|R^{b} f(x)\right|^{2}|x|^{2 \gamma} d x\right)^{1 / 2} \text {. }
$$

One computes easily that $|x|^{\gamma} \in A_{p 2} \Leftrightarrow-n / 2<\gamma<n / p^{\prime}$. For such $\gamma$, we can estimate

$$
\dot{B}_{22}^{\gamma}\left(|\xi|^{a} \hat{f}\right) \leqslant C\left\||f||x|^{\gamma}\right\|_{p}
$$


with $1 / 2=1 / p-b / n=1 / p+a / n$. An easy computation shows that $n / p^{\prime}=a$ $+n / 2$, and thus we conclude that

$$
\begin{gathered}
\dot{B}_{22}^{\gamma}\left(|\xi|^{a} \hat{f}\right) \leqslant C\left\||f||x|^{\gamma}\right\|_{p}, \\
-\frac{n}{2}<\gamma<a+\frac{n}{2}, \quad \frac{1}{2}=\frac{1}{p}+\frac{a}{n} .
\end{gathered}
$$

The second step is to observe that it suffices to prove Theorem 2.1 for a sequence of dimensions $N_{l} \rightarrow \infty$ as $l \rightarrow \infty$ because of the possibility of applying restriction theorems.

Proposition 2.3. If Theorem 2.1 holds for dimensions that are multiples of four, it holds for all dimensions.

Proof. Given a dimension $n$, choose $l$ so that $4 l \leqslant n<4 l+4$. Let $\psi$ be a $C_{0}^{\infty}$ function of the $(4 l+4-n)$ variables such that $\hat{\psi}(0)=1$. Given $f \in C_{c}^{m}\left(R^{n}\right)$, consider $F \in C_{c}^{m}\left(R^{4 l+4}\right)$ defined by

$$
F\left(\xi^{\prime}, \xi^{\prime \prime}\right)=f\left(\xi^{\prime}\right) \psi\left(\xi^{\prime \prime}\right)
$$

where $\xi^{\prime}$ are the variables in $R^{n}$ and $\xi^{\prime \prime}$ those in $R^{4 l+4-n}$. If we have proved the result for $R^{4 l+4}$, it follows that

$$
\left(\left|\xi^{\prime}\right|^{2}+\left|\xi^{\prime \prime}\right|^{2}\right)^{a / 2} \hat{F}\left(\xi^{\prime}, \xi^{\prime \prime}\right) \in \dot{B}_{22}^{\gamma}\left(R^{4 l+4}\right)
$$

for $0<\gamma<a+2 l+2$, since

$$
a+2 l+2 \geqslant a+\frac{n}{2}+(4 l+4-n) / 2 \geqslant\left(\frac{4 l+4-n}{2}\right) .
$$

The Fourier transform splits and hence

$$
\left(\left|\xi^{\prime}\right|^{2}+\left|\xi^{\prime \prime}\right|^{2}\right)^{a / 2} \hat{f}\left(\xi^{\prime}\right) \hat{\psi}\left(\xi^{\prime \prime}\right) \in \dot{B}_{22}^{\gamma}\left(R^{4 l+4}\right) .
$$

In particular, if $\gamma>(4 l+4-n) / 2$, we can restrict this function to $R^{n}$ and we obtain

$$
\hat{\psi}(0)\left|\xi^{\prime}\right|^{a} \hat{f}\left(\xi^{\prime}\right) \in \dot{B}_{22}^{\gamma-(4 l+4-n) / 2}\left(R^{n}\right) .
$$

As $\gamma$ ranges over $((4 l+4-n) / 2, a+2 l+2), \gamma-(4 l+4-n) / 2$ ranges over $(0, a+n / 2)$.

We will thus prove the theorem for $n=4 l, l \geqslant 1$. This restriction is convenient for the induction argument in the proof of Theorem 2.1, but again is not necessary.

Proof of Theorem 2.1. Note first that the theorem is trivial for $\lambda=2 k$, with $k$ an integer and $m=\lambda$.

The proof will be by induction over the ranges $2 k<\lambda<2 k+2$ and we first consider the case $0<\lambda<2$. In that case $-n / 2<\lambda-2<0$, and we can apply Proposition 2.2 by writing

$$
|\xi|^{\lambda} \hat{f}=|\xi|^{\lambda-2}|\xi|^{2} \hat{f}=-|\xi|^{\lambda-2} \widehat{\Delta f}
$$

Hence if $\Delta f \in L_{|x|^{\gamma}}^{p},|\xi|^{\lambda-2} \widehat{\Delta f} \in \dot{B}_{22}^{\gamma}$ for $-2 l<\gamma<(\lambda-2)+2 l$. If $f \in C_{c}^{2}$, this condition is satisfied. 
On the other hand, since

$$
\begin{aligned}
\Delta\left(|\xi|^{\lambda} \hat{f}\right) & =\lambda(\lambda+n-2)|\xi|^{\lambda-2} \hat{f}+\lambda|\xi|^{\lambda-2} \sum_{j=1}^{n} \xi_{j} \frac{\partial \hat{f}}{\partial \xi_{j}}+|\xi|^{\lambda} \Delta(\hat{f}) \\
& =\lambda(\lambda+n-2)|\xi|^{\lambda-2} \hat{f}-\lambda|\xi|^{\lambda-2} \sum_{j=1}^{n} \widehat{\frac{\partial}{\partial x_{j}}\left(x_{j} f\right)}-|\xi|^{\lambda} \widehat{|x|^{2} f}
\end{aligned}
$$

we note that if $\lambda-2+2 l \leqslant \gamma<\lambda+2 l, \gamma-2$ satisfies the conditions of the previous paragraph and hence if $f \in C_{c}^{2},\left(\partial / \partial x_{j}\right)\left(x_{j} f\right) \in C_{c}^{2},|x|^{2} f \in C_{c}$, we conclude that

$$
\Delta\left(|\xi|^{\lambda} \hat{f}\right) \in \dot{B}_{22}^{\gamma-2}\left(R^{4 l}\right)
$$

i.e., $|\xi|^{\lambda} \hat{f} \in \dot{B}_{22}^{\gamma}\left(R^{4 l}\right)$. This completes the proof that if $0<\lambda<2,-2 l<\gamma<\lambda+2 l$, $|\xi|^{\lambda} \hat{f} \in \dot{B}_{22}^{\gamma}\left(R^{4 l}\right)$, and we require that $f \in C_{c}^{3}\left(R^{4 l}\right)$.

Suppose we have shown that if $2 j-2<\lambda \leqslant 2 j, f \in C_{c}^{2 j+1},-2 l<\gamma<\lambda+2 l$, $|\xi|^{\lambda} \hat{f} \in \dot{B}_{22}^{\gamma}\left(R^{4 l}\right)$, i.e., that the statement of the theorem is valid for $(j-1)$. We will show that it holds for $j$. Suppose $2 j<\lambda \leqslant 2 j+2$. For $\lambda=2 j+2$, there is nothing to prove. If $2 j<\lambda<2 j+2$, then if $\Delta f \in C_{c}^{2 j+1},|\xi|^{\lambda} \hat{f}=-|\xi|^{\lambda-2} \Delta f \in \dot{B}_{22}^{\gamma}$ if $-2 l<$ $\gamma<(\lambda-2)+2 l$.

Moreover, if $\lambda-2+2 l \leqslant \gamma<\lambda+2 l, \gamma-2>0$ and using (2.1), $\Delta\left(|\xi|^{\lambda} \hat{f}\right) \in$ $\dot{B}_{22}^{\gamma-2}\left(R^{4 l}\right)$ which implies $|\xi|^{\lambda} \hat{f} \in \dot{B}_{22}^{\gamma}\left(R^{4 l}\right)$.

The only requirement is that $\Delta f \in C_{c}^{2 j+1}, f \in C_{c}^{2 j+1},\left(\partial / \partial x_{j}\right)\left(x_{j} f\right) \in C_{c}^{2 j+1}$, $|x|^{2} f \in C_{c}^{2 j+1}$, which will be fulfilled if $f \in C_{c}^{2 j+3}$.

This proves the theorem for $n=4 l$. An appeal to Proposition 2.2 gives the general result.

REMARKs. (1) Note that we actually showed that if $n=4 l,-n / 2<\gamma<\lambda+$ $n / 2, \lambda+n / 2>0, m \geqslant \max (2([\lambda / 2]+2), 0)$, then if $f \in C_{c}^{m},|\xi|^{\lambda} \hat{f} \in \dot{B}_{22}^{\gamma}\left(R^{4 l}\right)$. The restriction to $\gamma>0$ in the final result is only because of the appeal to Proposition 2.2. Slightly more work would have given the result in this generality. It is also clear that at the expense of more effort we could have kept track of the precise weighted $L^{p}$ estimates on $\Delta^{k} f$ required for the theorem.

Corollary 2.4. For $s>0$, if $\widehat{\Delta^{s} f}(\xi)=|\xi|^{2 s} \hat{f}(\xi), m>2 s+n / 2$, and if $f \in$ $C_{c}^{m}\left(R^{n}\right), \widehat{\Delta^{s f}} \in \dot{B}_{21}^{n / 2}\left(R^{n}\right) \subseteq F L^{1}\left(R^{n}\right)$.

Proof. For $s>0,|\xi|^{2 s} \hat{f} \in L^{2}\left(R^{n}\right)$ if $m>2 s+n / 2$. By Theorem 2.1, if $m>2[s]$ $+2,|\xi|^{s} \hat{f} \in \dot{B}_{22}^{\gamma}\left(R^{n}\right)$ for any $0<\gamma<s+n / 2$. Since $s>0$, we can take a $n / 2<\gamma$ $<2 s+n / 2$, and then by Theorem 2.1 , we conclude

$$
|\xi|^{2 s} \hat{f} \in \dot{B}_{21}^{n / 2}\left(R^{n}\right) \subseteq F L^{1}
$$

This completes the proof.

Corollary 2.5. If $0<\gamma<b+n / 2+|\alpha|, b+2|\alpha|+n / 2>0, b+2|\alpha| \neq 0$, $|\xi|^{b} \xi^{\alpha} \hat{f} \in \dot{B}_{22}^{\gamma}$ 
Proof. Induction on $|\alpha|$. The result is certainly true for $|\alpha|=0$. Let us also check it for $|\alpha|=1$. Observe that

$$
\begin{gathered}
\partial_{\xi}\left(|\xi|^{a} \hat{f}\right)=a|\xi|^{a-2} \xi_{j} \hat{f}+|\xi|^{a} \partial_{\xi} \hat{f} \\
\Rightarrow(\lambda+2)|\xi|^{\lambda} \xi_{j} \hat{f}=\partial_{\xi}\left(|\xi|^{\lambda+2} \hat{f}\right)-|\xi|^{\lambda+2} \partial_{\xi} \hat{f} \\
=\partial_{\xi_{j}}\left(|\xi|^{\lambda+2} \hat{f}\right)+i|\xi|^{\lambda+2} \widehat{x_{j} f} .
\end{gathered}
$$

Suppose $0<\gamma<b+n / 2+1, b+2+n / 2>0$. Then $0<\gamma+1<b+2+$ $n / 2 \Rightarrow|\xi|^{b+2} \hat{f} \in \dot{B}_{22}^{\gamma+1}$ if $f \in C_{c}^{m}, m \geqslant 2([(b+2) / 2]+2)$ and $|\xi|^{b+2} \widehat{x_{j} f} \in \dot{B}_{22}^{\gamma}$ and if $b+2 \neq 0,|\xi|^{b} \xi_{j} \hat{f} \in \dot{B}_{22}^{\gamma}$.

Assume the result has been proved for $|\alpha|=m$. A similar argument to that used previously proves the result for $|\alpha|=m+1$ !

COROllary 2.6. If $s>-|\alpha|, \Delta^{s}\left(D^{\alpha} f\right) \in L^{1}$.

ProOF. $|\xi|^{2 s} \widehat{D^{\alpha} f} \in \dot{B}_{22}^{\gamma} \cap L^{2} \subseteq \dot{B}_{21}^{n / 2} \subset F L^{1}$, if

$$
n / 2<2 s+n / 2+|\alpha| \text {, i.e., } s>-|\alpha| \text {. }
$$

With a little more care, we can also give a weight.

Corollary 2.7. If $s>0, \rho<s,\left(1+|x|^{2 \rho}\right) \Delta^{s} f \in L^{1}$.

Proof. We want to show that $|x|^{2 \rho} \Delta^{s} f \in L^{1}$, or equivalently, that $\Delta^{\rho}\left(\widehat{\Delta^{s} f}\right) \in F L^{1}$. It suffices to show that $\Delta^{\rho}\left(\widehat{\left.\Delta^{s} f\right)} \in \dot{B}_{21}^{n / 2}\right.$. Since $\widehat{\Delta^{s} f}=|\xi|^{2 s} \hat{f}$, we apply Theorem 2.1 and the remark before that $\widehat{\Delta^{s} f} \in \dot{B}_{22}^{\gamma}$, for any $\gamma<2 s+n / 2$, and correspondingly $\Delta^{\rho}\left(\widehat{\Delta^{s} f}\right) \in \dot{B}_{21}^{\gamma-2 \rho}$, which can be taken as $\dot{B}_{21}^{n / 2}$ as long as $2(s-\rho)+n / 2>n / 2$, i.e., $s-\rho>0$.

3. An embedding theorem with applications to the study of Fourier multipliers for weighted $L^{2}$ spaces. The estimates of $\$ 2$ suggest that there is a close relation between the $\dot{B}_{22}^{\gamma}$ norm of a function and a weighted $L^{2}$ norm of a function. Using a form of Pitt's theorem due to Flett [1], we will prove a quantitative form of this and discuss its application to an embedding theorem.

TheOREM 3.1. Suppose $-n / 2<\gamma \leqslant 0$. Then

$$
L_{|x|^{-2 \gamma}}^{2} \rightarrow \dot{B}_{22}^{\gamma}
$$

and

$$
\|f\|_{\dot{B}_{1_{2}}} \leqslant A\|f\|_{L_{|x|}^{2}-2 \gamma}
$$

Proof. Observe that, by Herz [2],

$$
\|f\|_{\dot{B}_{z_{2}}} \cong\|\hat{f}\|_{K \gamma_{2}}=\left(\int|x|^{2 \gamma}|\hat{f}(x)|^{2} d x\right)^{1 / 2}=\left\||x|^{\gamma} \hat{f}\right\|_{2} .
$$

We apply Theorem 4(iv) of Flett [1], with $p=q=2=c$ and $\varphi=\psi=|x|^{n}$. Since we must have $1<r \leqslant 2$, if we write $\gamma=-a, 0<a<n / 2(a=0$ is trivial), the result of Flett

$$
\left\|\hat{f}\left(|x|^{n}\right)^{1 / r^{\prime}-1 / 2}\right\|_{2} \leqslant B\left\|f\left(|x|^{n}\right)^{1 / r-1 / 2}\right\|_{2}
$$


can be applied if $n\left(1 / r^{\prime}-1 / 2\right)=-a$. For this choice $1 / r=1 / 2+a / n$, and one verifies easily that $1<r \leqslant 2$, and we obtain the estimate

$$
\|f\|_{B_{y_{2}}} \leqslant A\left\|\hat{f}\left(|x|^{n}\right)^{1 / r^{\prime}-1 / 2}\right\|_{2} \leqslant A B\left\|f\left(|x|^{n}\right)^{1 / r-1 / 2}\right\|_{2} \leqslant A B\left\|f|x|^{a}\right\|_{2} .
$$

THEOREM 3.2. Suppose $0 \leqslant \gamma<n / 2$. We have the embedding

$$
\dot{B}_{22}^{\gamma} \rightarrow L_{|x|^{-2 \gamma}}^{2},
$$

and there exists a constant such that

$$
\|f\|_{L_{|x|}^{2}-2 \gamma} \leqslant A\|f\|_{\dot{B}_{22}^{\prime}} .
$$

This follows from duality applied to Theorem 3.1. This embedding should be compared with the standard embedding $\dot{B}_{22}^{\gamma} \rightarrow L^{p, 2}, 1 / p=1 / 2-\gamma / n, 0<\gamma<$ $n / 2$. Our next result shows that Theorem 3.2 is a corollary of this result.

THEOREM 3.3. $L^{p, 2} \rightarrow L_{|x|^{-2 \alpha}}^{2}, 1 / p=1 / 2-\alpha / n, 0 \leqslant \alpha<n / 2$.

Proof. This will be accomplished in three steps. Note first that $L_{|x|^{2 \alpha}}^{\infty} \rightarrow L^{q, \infty}$, $q=n / 2 \alpha$, since if $\Phi(x)|x|^{2 \alpha} \in L^{\infty}$,

$$
|\Phi(x)| \leqslant M|x|^{-2 \alpha}, \quad \text { a.e., }
$$

and then, since $|x|^{-2 \alpha} \in L^{n / 2 \alpha, \infty}$, we are done. It now follows from duality that

$$
L^{p, 1} \rightarrow L_{|x|^{-2 \alpha}}^{1}, \quad 1 / p=1-2 \alpha / n .
$$

Indeed, if $g \in L^{p, 1}, f \in L_{|x|^{-2 \alpha}}^{\infty},\|f\| \leqslant 1$, then

$$
\begin{aligned}
\|g\|_{L_{|x|}^{1}-2 \alpha} & =\sup _{f}\left|\int g(x) f(x) d x\right| \leqslant A\|g\|_{p, 1}\|f\|_{n / 2 \alpha, \infty} \\
& \leqslant A B\|g\|_{p, 1}\|f\| \leqslant A B\|g\|_{p, 1} .
\end{aligned}
$$

Finally, if $f \in L^{p, 2},|f|^{2} \in L^{p / 2,1}$ and thus $|f|^{2} \in L_{|x|^{-2 \alpha}}^{1}$, with $2 / p=1-2 \alpha / n$, and hence, $|f| \in L_{|x|^{-2 \alpha}}^{2}, 1 / p=1 / 2-\alpha / n$.

REMARK. 1. Given a weight $|x|^{\gamma}, \gamma>0$, the prior argument shows that $L_{|x|^{\gamma}}^{\infty} \subseteq$ $L^{n / \gamma, \infty}$, and then by duality

$$
L^{r, 1} \rightarrow L_{|x|^{-\gamma}}^{1}, \quad 1 / r+\gamma / n=1,
$$

and consequently,

$$
L^{q p} \rightarrow L_{|x|^{-\gamma p}}^{p}, \quad 1 / q=1 / p-\gamma / n .
$$

This last follows since $f \in L^{q p}$ implies $|f|^{p} \in L^{q / p, 1} \subseteq L_{|x|^{-p}}^{1}$, if $p / q+\gamma p / n=1$.

2. As will be noted in $\S 4$, Theorem 3.1 is more convenient for use in the study of hyperbolic polynomials.

As a preview of the technique to be used in the proof of our main theorem, we give an alternate proof of Corollary 2.4 .

Proposition 3.4. Suppose $-n / 2<\gamma<0, \lambda<\gamma+n / 2$; then

$$
\left\||\xi|^{\gamma} \hat{f}\right\|_{\dot{B}_{22}^{\gamma}} \leqslant A\left\||\xi|^{\lambda-\gamma} \hat{f}\right\|_{2} \text {. }
$$

The $L^{2}$ norm involved in the last integral is finite for any $f \in C_{c}^{m}$ with $m$ large enough and $\lambda-\gamma>-n / 2$. 
As the remarks before the proof of Theorem 2.1 show, it is enough to show that for any $\lambda>0,|\xi|^{\lambda} \hat{f} \in \dot{B}_{22}^{n / 2+\varepsilon}$. An easy computation gives

$$
\Delta\left(|\xi|^{\lambda} \hat{f}\right)=C|\xi|^{\lambda-2} \hat{f}+2\left(\sum_{j=1}^{n} \xi_{j} \partial_{\xi} \hat{f}\right)|\xi|^{\lambda-2}+|\xi|^{\lambda} \Delta \hat{f}
$$

and an inductive argument shows that for $\Delta^{l+1}(|\xi| \lambda \hat{f})$, all the terms have powers of $\xi$ exceeding $\lambda-2 l-2$. By Proposition 2.2 , these terms will belong to $\dot{B}_{22}^{\ell-2}(0<\varepsilon<$ 2) if

$$
\lambda-\gamma=(\lambda-2 l-2)-(\varepsilon-2)>-2 l=-n / 2,
$$

i.e., if $\lambda>\varepsilon$. We also have $-n / 2<\varepsilon-2<0$ since $n \geq 4$. Given $\lambda>0$, choose $0<\varepsilon<2, \varepsilon<\lambda$ and we obtain

$$
\Delta^{l+1}\left(|\xi|^{\lambda} \hat{f}\right) \in \dot{B}_{22}^{\varepsilon-2}
$$

which implies that

$$
|\xi|^{\lambda} \hat{f} \in \dot{B}_{22}^{2 l+\varepsilon}=\dot{B}_{22}^{n / 2+\varepsilon} .
$$

In the study of the hyperbolic $P$, we shall be forced to use nonintegral powers of $\Delta$.

While the main thrust of our paper has been to give sufficient conditions to guarantee that $|P(\xi)|^{\lambda} \hat{f} \in F L^{1}$, we can also give sufficient conditions to guarantee that $|P(\xi)|^{\lambda} \hat{f} \in \dot{B}_{p 1}^{n / p}$. At this point we make some remarks on $|\xi|^{\lambda} \hat{f}$. To prove that it belongs to $\dot{B}_{p 1}^{n / p}$ it suffices to show that $|\xi|^{\lambda} \hat{f} \in L^{p} \cap \dot{B}_{p p}^{n / p+\varepsilon}$. As long as $\lambda>-n / p$, $|\xi|^{\lambda} \hat{f} \in L^{p}$. To prove that $|\xi|^{\lambda} \hat{f} \in \dot{B}_{p p}^{n / p+\varepsilon}$, it suffices to prove an embedding theorem.

THEOREM 3.5. $L_{\mid x p^{p^{\prime} \gamma}} \subseteq \dot{B}_{p p}^{-\gamma}, 1<p<\infty$.

Proof. By duality, it suffices to prove that

$$
\dot{B}_{p p}^{\gamma} \subseteq L_{|x| p r}^{p}, \quad 1<p<\infty .
$$

The classical embedding theorem implies that

$$
\dot{B}_{p p}^{\gamma} \subseteq L^{q, p}, \quad 1 / q=1 / p-\gamma / n,
$$

and the remark after Theorem 3.2 shows that

$$
L^{q, p} \subseteq L_{|x|^{-p \gamma}}^{p}
$$

which gives the result.

We postpone the proof of the result for $|\xi| \hat{f}$ until the tools have been set out in $\S 4$.

4 The wave polynomial. In this section, we consider the case in which $P(\xi)=\xi_{1}^{2}$ $+\cdots+\xi_{p}^{2}-\xi_{p+1}^{2}-\cdots-\xi_{n}^{2}$. The characteristic set for this function is a conic in $R^{n}$ called the light cone and denoted here by $\Gamma$. Many of our integrals will be split according to their distances from the light cone. Since $\Gamma=\{\xi \mid P(\xi)=0\}$, if we write a point $\xi \in R^{n}$ as $\xi=\left(\xi^{\prime}, \xi^{\prime \prime}\right)$, where $\xi^{\prime}=\left(\xi_{1}, \ldots, \xi_{p}\right), \xi^{\prime \prime}=\left(\xi_{p+1}, \ldots, \xi_{n}\right)$ and if we set $r=\left|\xi^{\prime}\right|, s=\left|\xi^{\prime \prime}\right|$, then it is easily seen that

$$
d(\xi, \Gamma)=\inf \{|\xi-\eta|: \eta \in \Gamma\}=|r-s| / \sqrt{2} .
$$


Once again our strategy will be to show that $|P(\xi)|^{\lambda} \hat{f}(\xi) \in \dot{B}_{21}^{n / 2}$, for suitable restrictions on $\lambda$, and the key step will be in deciding for which $\lambda,|P(\xi)|^{\lambda} \hat{f}(\xi) \in \dot{B}_{22}^{\gamma}$ $\cap L^{2}$. The finiteness of a weighted $L^{2}$ norm of a fractional power will give the appropriate result. Note that we may assume $n \geqslant 2$ since $P$ is elliptic for $n=1$; we may also assume $p \geqslant 1, q \geqslant 1$. (If only the question of integrability of the Fourier transform were being considered, we could assume $n \geqslant 3$ because a linear change of variables when $n=2$ reduces $P(\xi)$ to $\eta_{1} \eta_{2}$ and two successive applications of the one dimensional results show that $|P(\xi)|^{\lambda} \hat{f}(\xi) \in A\left(R^{2}\right)$ for any $\lambda>0$.)

TheOREM 4.1. If $f \in C_{c}^{m}, m>4(|\lambda|+\gamma)+n, \lambda>-1 / 2, \gamma>0$,

$$
\int|\hat{f}(\xi)|^{2}|P(\xi)|^{2 \lambda}|\xi|^{2 \gamma} d \xi<+\infty
$$

Proof. Without loss of generality we may assume $\lambda<0$ since $|P(\xi)| \leqslant|\xi|^{2}$ and the conditions suffice in the elliptic case. For $\lambda<0$, let $\lambda=-b, 0<b<1 / 2$.

Split the integral

$$
\int|\hat{f}(\xi)|^{2}|P(\xi)|^{2 \lambda} d \xi=\int_{d(\xi, \Gamma)<1 / \sqrt{2}}+\int_{d(\xi, \Gamma)>1 / \sqrt{2}}
$$

into a piece near the light cone and a piece away from the light cone. Away from the light cone,

$$
|P(\xi)|=|(r+s)(r-s)| \geqslant|r+s| \geqslant|\xi|,
$$

which allows us to estimate

$$
\int_{d(\xi, \Gamma)>1 / \sqrt{2}}\left(|\hat{f}(\xi)|^{2} /|P(\xi)|^{2 b}\right)|\xi|^{2 \gamma} d \xi \leqslant A \int_{d(\xi, \Gamma)>1 / \sqrt{2}}|\hat{f}(\xi)|^{2}|\xi|^{2 \gamma-2 b} d \xi<+\infty,
$$

since $\gamma-b>-n / 2$ is valid for $n \geqslant 2$. Near the light cone, we appeal to the following result.

LEMMA 4.2. If $0<b<1 / 2, m>4 b+n$,

$$
\int_{d(\xi, \Gamma)<1}|P(\xi)|^{-2 b}\left(1+|\xi|^{2}\right)^{-m / 2} d \xi<+\infty .
$$

Proof. Rewrite the integral in polar coordinates

$$
\iint\left|r^{2}-s^{2}\right|^{-2 b}\left(1+r^{2}+s^{2}\right)^{-m / 2} r^{p-1} d r s^{q-1} d s,
$$

split this into two pieces which are handled similarly,

$$
\iint=\iint_{0<r-s<\sqrt{2}}+\iint_{0<s-r<1}=K_{1}+K_{2} .
$$

Rewrite $K_{1}$ as an iterated integral

$$
\begin{aligned}
K_{1} & =\int_{0}^{\infty}\left(\int_{s}^{s+\sqrt{2}}\left|r^{2}-s^{2}\right|^{-2 b}\left(1+r^{2}+s^{2}\right)^{-m / 2} r^{p-1} d r\right) s^{q-1} d s \\
& =\int_{0}^{\sqrt{2}}\left(\int_{s} s+\sqrt{2}\right)+\int_{\sqrt{2}}^{\infty}\left(\int_{s} s+\sqrt{2}\right)=K_{11}+K_{12} .
\end{aligned}
$$


Let $\Psi(s)=\int_{s}^{s+\sqrt{2}}\left|r^{2}-s^{2}\right|^{-2 b}\left(1+r^{2}+s^{2}\right)^{-m / 2} r^{p-1} d r$. In the integral involving $K_{11}$, since $s \leqslant r \leqslant s+\sqrt{2}$,

$$
1 / \sqrt{2} \leqslant \frac{\sqrt{1+r^{2}+s^{2}}}{1+s}<\sqrt{2} \text {, }
$$

and we can estimate

$$
\Psi(s) \leqslant \frac{(s+\sqrt{2})^{p-1}}{(s+\sqrt{2})^{m}(2 s)^{2 b}} \int_{s}^{s+\sqrt{2}} \frac{d r}{(r-s)^{2 b}},
$$

and the integral converges since $b<1 / 2$, yielding the estimate

$$
\Psi(s) \leqslant A \frac{(s+\sqrt{2})^{p-m-1}}{s^{2 b}} ;
$$

and consequently

$$
K_{11}<\int_{0}^{1} \Psi(s) s^{q-1} d s \leqslant A \int_{0}^{1} s^{q-2 b-1} d s<+\infty,
$$

since $q \geqslant 1>2 b$.

For $K_{12}$, we first change variables $r=s u$ and rewrite

$$
\begin{aligned}
K_{12} & \leqslant C \int_{\sqrt{2}}^{\infty}\left(\int_{s}^{s+\sqrt{2}} \frac{r^{p-1}}{\left(1+r^{2}+s^{2}\right)^{m / 2}(r+s)^{2 b}(r-s)^{2 b}}\right) s^{q-1} d s \\
& =C \int_{\sqrt{2}}^{\infty}\left(\int_{1}^{1+\sqrt{2} / s} \frac{s^{p-1} u^{p-1} s d u}{\left(1+\left(u^{2}+1\right) s^{2}\right)^{m / 2}(1+u)^{2 b} s^{2 b}(1-u)^{2 b} s^{2 b}}\right) s^{q-1} d s \\
& \leqslant C \int_{\sqrt{2}}^{\infty}\left(\int_{1}^{1+\sqrt{2} / s} \frac{u^{p-1} d u}{(1+u)^{m+2 b}(1-u)^{2 b}}\right) s^{p+q-1-4 b-m} d s .
\end{aligned}
$$

Since $s \geqslant \sqrt{2}$, the $u$-integral is bounded by

$$
\int_{1}^{2} \frac{u^{p-1} d u}{(1+u)^{2 b+m}(1-u)^{2 b}}
$$

which converges since $b<1 / 2$, and we have the estimate

$$
K_{12} \leqslant C \int_{\sqrt{2}}^{\infty} s^{n-4 b-m-1} d s,
$$

which converges since $n-4 b-m<0$.

REMARK. The same argument shows that

$$
\int_{d(\xi, \Gamma)<\min \left(\left|\xi^{\prime}\right|,\left|\xi^{\prime \prime}\right|\right)} \frac{1}{|P(\xi)|^{2 b}\left(1+|\xi|^{2}\right)^{m / 2}} d \xi<+\infty,
$$

under the same conditions. The inner integral in $K_{1}$ becomes

$$
\Psi_{1}(s)=\int_{s}^{2 s} \frac{r^{p-1}}{\left(r^{2}-s^{2}\right)^{2 b}\left(1+r^{2}+s^{2}\right)^{n b / 2}} d r
$$

which is estimated as before. 
The end of the proof of Theorem 4.1 then follows easily since if $f \in C_{c}^{m}$,

$$
|\hat{f}(\xi)| \leqslant C_{m}\left(1+|\xi|^{2}\right)^{-m / 2}
$$

and since $|\xi|^{\gamma} \leqslant\left(1+|\xi|^{2}\right)^{\gamma / 2}$.

Remarks. (1) The result was valid in the elliptic case for $\lambda>-n / 2$. The dimunition of the range of $\lambda$ will lead to corresponding problems in our later analysis.

(2) The estimates are similar to those used by Fefferman, cited in [4]. What Fefferman shows is that if $\lambda \leqslant-1 / 2, \hat{f}(0) \neq 0,|P(\xi)|^{\lambda} \hat{f} \notin L^{2}$ and that $F^{-1}\left(|P(\xi)|^{\lambda} \hat{f}\right)$ $\notin L^{1}$ if $\lambda \leqslant n / 2-1$.

(3) The singularity is given by $r-s$, which allows us to prove that if $\lambda>-1$, $d(\xi, \Gamma)^{\lambda} \hat{f} \in L^{2}$, if $m>2 \lambda+n$.

Now we are in a position to apply the embedding theorem (Theorem 3.1).

Corollary 4.3. Suppose $0 \leqslant \gamma<n / 2, \lambda>-1 / 2$; then

$$
|P(\xi)|^{\lambda} \hat{f} \in \dot{B}_{22}^{-\gamma}
$$

if $f \in C_{c}^{m}, m$ large enough.

The key point here is that $\gamma$ may be chosen arbitrarily small and positive. However, if we try to construct a proof like that given in $\$ 3$, we run into two problems. Since our results depend on dimension, it does not suffice to prove the theorem for dimensions that are multiples of four. Moreover, the natural differential operator to apply, $P(D)=D_{1}^{2}+\cdots+D_{p}^{2}-D_{p+1}^{2}-\cdots-D_{n}^{2}$, does not shift the Besov spaces like the Laplacian, while the Laplacian eats too many powers of $P(\xi)$. We first consider the effect of applying the Laplacian once.

COROllary 4.4. Suppose $0 \leqslant \gamma<n / 2, \lambda>3 / 2$. Then, if $m$ is large enough

$$
|P(\xi)|^{\lambda} \hat{f} \in \dot{B}_{22}^{2-\gamma}
$$

for any $f \in C_{c}^{m}\left(R^{n}\right)$.

Proof. An easy computation shows that $\Delta\left(|P(\xi)|^{\lambda} \hat{f}\right)$ is a sum of terms in $L_{|x|^{2}}^{2}$, since, off the light cone,

$$
\begin{aligned}
\Delta\left(|P(\xi)|^{\lambda} \hat{f}\right)= & \Delta\left(|P(\xi)|^{\lambda}\right) \hat{f}(\xi)+2 \nabla\left(|P(\xi)|^{\lambda}\right) \cdot \nabla \hat{f}(\xi)+|P(\xi)|^{\lambda} \Delta \hat{f} \\
= & 4 \lambda(\lambda-2)|P(\xi)|^{\lambda-2}|\xi|^{2} \hat{f}+4 \lambda|P(\xi)|^{\lambda-2} \hat{f}(\xi)|\xi|^{2} \\
& +2 \lambda(p-q)|P(\xi)|^{\lambda-2} P(\xi) \hat{f} \\
& +4 \lambda\left(\sum_{j=1}^{n} \varepsilon_{j} \xi \hat{f}(\xi)\right)|P(\xi)|^{\lambda-2} P(\xi)+|P(\xi)|^{\lambda} \Delta \hat{f}
\end{aligned}
$$

where $\varepsilon_{j}=+1, j=1, \ldots, p, \varepsilon_{j}=-1$, for $j=p+1, \ldots, n$. Notice now that $p(\xi) \hat{f}(\xi)=\widehat{p(D) f}(\xi)$ and $p(D) \hat{f}(\xi)=\widehat{p(x) f(x)}(\xi)$ and both are Fourier transforms of $C_{c}^{m}$ functions. Every term above contains at least $|P(\xi)|^{\lambda-2}$, hence, if $\lambda-2>$ $-1 / 2$, all terms are in $L_{|x|^{2 \gamma}}^{2}$ and so in $\dot{B}_{22}^{-\gamma}$. This concludes the proof.

If one attempts to apply this result to show that $|P(\xi)|^{\lambda} \hat{f} \in \dot{B}_{2}^{n} /{ }^{2}$ in dimensions 3 or 4 , we see that it fails in dimension 4 since $2-\gamma<n / 2$, and in dimension 3 , we 
obtain that if $\lambda>3 / 2,|P(\xi)|^{\lambda} \hat{f} \in \dot{B}_{21}^{3 / 2}$. What we need is the fractional analogue of Corollaries 4.4 and 4.5 . Corollary 4.4 amounts to a result about $\Delta^{0}$, and 4.5 to a result about $\Delta^{1}$. Interpolation gives the next result.

THEOREM 4.5. If $\lambda>2 \rho-1 / 2,0 \leqslant \gamma<n / 2, f \in C_{c}^{m}, m$ large,

$$
\Delta^{\rho}\left(|P(\xi)|^{\lambda} \hat{f}\right) \in \dot{B}_{22}^{-\gamma} .
$$

Proof. It suffices to prove this for $0<\rho<1$ since we can always write $\rho=K+\theta, \quad 0<\theta<1$, and a tedious computation shows that the terms in $\Delta^{K}\left(|P(\xi)|^{\lambda} \hat{f}\right)$ all have $P(\xi)$ to at least the power $\lambda-2 K$. To prove it in the range $0<\rho<1$, we will use complex interpolation. We therefore postpone the proof to note the immediate corollary.

TheOREM 4.6. Suppose $\lambda>n / 2-1 / 2, f \in C_{c}^{m}, m$ large; then

$$
|P(\xi)|^{\lambda} \hat{f} \in \dot{B}_{21}^{n / 2} \text {. }
$$

Proof. Consider $\rho=n / 4+\varepsilon^{\prime}$ where $\varepsilon^{\prime}$ is small enough that $\lambda-2 \rho>-1 / 2$. By Theorem 4.6, $\Delta^{\rho}\left(|P(\xi)|^{\lambda} \hat{f}\right) \in \dot{B}_{22}^{-\delta}$ for any $\delta>0$. Hence, $|P(\xi)|^{\lambda} \hat{f} \in \dot{B}_{22}^{n / 2+2 e^{\prime}-\delta}$, and if we choose $\delta<2 \varepsilon^{\prime},|P(\xi)|^{\lambda} \hat{f} \in \dot{B}_{22}^{s}$ for some $s>n / 2$. Since $|P(\xi)|^{\lambda} \hat{f} \in L^{2}$ for any $\lambda>-1 / 2$, the result of Herz implies that $|P(\xi)|^{\lambda} \hat{f} \in \dot{B}_{21}^{n / 2}$.

There remains only the complex interpolation to conclude the proof of Theorem 4.6. Fix a $\lambda_{1}>-1 / 2, \lambda_{2}>3 / 2$, and $0 \leqslant \gamma<n / 2$. We will consider the following family of operators,

$$
T_{z} f=R^{\gamma}\left(\Delta^{z}\left(|P(\xi)|^{\lambda_{1}(1-z)+\lambda_{2} z} \hat{f}\right)\right), \quad 0 \leqslant \operatorname{Re} z<1 .
$$

Notice that when $\operatorname{Re} z=0$,

$$
T_{i t} f=R^{\gamma}\left(\Delta^{i t}\left(|P(\xi)|^{\lambda_{1}}|P(\xi)|^{\left(\lambda_{2}-\lambda\right) i t} \hat{f}\right)\right)
$$

will behave like $R^{\gamma}\left(|P(\xi)|^{\lambda_{1}} \hat{f}\right) \in L^{2}$, while when $\operatorname{Re} z=1$,

$$
T_{1+i \hbar} f=R^{\gamma}\left(\Delta^{i t}\left(\Delta\left(|P(\xi)|^{\lambda_{2}}|P(\xi)|^{\left(\lambda_{1}-\lambda_{2}\right) i t} \hat{f}\right)\right)\right)
$$

will behave like $R^{\gamma}\left(\Delta\left(|P(\xi)|^{\lambda_{2} f}\right)\right) \in L^{2}$. If the family depends analytically on $z$ in the sense of Stein's theorem, we conclude that $T_{\theta} f \in L^{2}, 0<\theta<1$, i.e., that

$$
\begin{gathered}
R^{\gamma}\left(\Delta^{\theta}\left(|P(\xi)|^{\lambda_{1}(1-\theta)+\lambda_{2} \theta} \hat{f}\right)\right) \in L^{2}, \\
\Delta^{\theta}\left(|P(\xi)|^{\lambda_{1}(1-\theta)+\lambda_{2} \theta} \hat{f}\right) \in \dot{B}_{22}^{-\gamma},
\end{gathered}
$$

and as $\lambda_{1}, \lambda_{2}$ range over all possible choices, $\lambda_{1}(1-\theta)+\lambda_{2} \theta$ ranges over all numbers subject to

$$
\lambda_{1}(1-\theta)+\lambda_{2} \theta>\left(-\frac{1}{2}\right)(1-\theta)+\theta\left(\frac{3}{2}\right)=2 \theta-\frac{1}{2} .
$$

The analyticity of $z \rightarrow \Delta^{z} \varphi$ is well known for $0<\operatorname{Re} z<1$, while that of $z \rightarrow$ $|P(\xi)|^{\lambda_{1}(1-z)+\lambda_{2} z \hat{f}}$ is obvious. All that remains is to observe that $R^{\gamma}$ and $\Delta^{i t}$ commute and that $\Delta^{i t}: L^{2} \rightarrow L^{2}$, and that the weighted $L^{2}$ norm of $|P(\xi)|^{ \pm\left(\lambda_{1}-\lambda_{2}\right) i t \hat{f}}$ is the same as that of $\hat{f}$.

We conclude with two general results giving sufficient conditions guaranteeing that $|\xi|^{\lambda} \hat{f}$ (resp. $|P(\xi)|^{\lambda} \hat{f}$ ) belongs to $\dot{B}_{p 1}^{n / p}$. 
THEOREM 4.7. For any $\lambda>0,1<p<\infty,|\xi|^{\lambda} \hat{f}(\xi) \in \dot{B}_{p 1}^{n / p}$, if $f \in C_{c}^{m}$, with $m$ large enough.

THEOREM 4.8. For $\lambda>(n-1) / p, 1<p<\infty,|P(\xi)|^{\lambda} \hat{f}(\xi) \in \dot{B}_{p 1}^{n / p}$, if $f \in C_{c}^{m}$, with $m$ large enough.

Both results follow from the same types of computations. As we remarked earlier, $|\xi|^{\lambda} \hat{f} \in L^{p}$ if $\lambda>-n / p$. To apply Theorem 3.4, we must also verify that, for any $\varepsilon>0,|\xi|^{\lambda} \hat{f} \in L_{|x|^{p^{\prime} e}}^{e}$, i.e., the finiteness of the integral

$$
\left.\left.\int|| \xi\right|^{\lambda} \hat{f}(\xi)\right|^{p}|\xi|^{p^{\prime} \varepsilon} d \xi=\int|\xi|^{\lambda p+p^{\prime} \varepsilon}|\hat{f}(\xi)|^{p} d \xi,
$$

and this last integral is finite if $\lambda p+p^{\prime} \varepsilon>-n$. Applying the appropriate version of Theorem 4.6, we see that $\Delta^{\rho}\left(|\xi|^{\lambda} \hat{f}\right) \in \dot{B}_{p p}^{-\varepsilon}$ if $(\lambda-2 \rho) p+p^{\prime} \varepsilon>-n$, and if we choose $2 \rho=n / p+\delta$, we obtain that if $\lambda p-p \delta+p^{\prime} \varepsilon>0$

$$
|\xi|^{\lambda} \hat{f} \in \dot{B}_{p p}^{n / p+\delta-\varepsilon} \text {. }
$$

The appropriate sequence of steps is to choose $\varepsilon>0, \delta>0$ both small with $\delta>\varepsilon$. Then for $\lambda>\delta-\varepsilon /(p-1)$,

$$
|\xi|^{\lambda} \hat{f} \in \dot{B}_{p 1}^{n / p}
$$

In the hyperbolic case, $|P(\xi)|^{\lambda} \hat{f} \in L^{p}$ if $\lambda>-1 / p$, and $|P(\xi)|^{\lambda} \hat{f} \in L_{\left.x\right|^{p^{\prime} e}}$ if $\lambda>$ $-1 / p$. Making the same choice of $\rho$, we obtain Theorem 4.8.

When $p=2$, Theorem 4.8 gives the same result as Theorem 4.6. For $p<2$ it gives a sharper result since $\dot{B}_{p 1}^{n / p} \subseteq \dot{B}_{21}^{n / 2}$ then. For $p>2$ it gives less but since $(n-1) / p \rightarrow 0$ as $p \rightarrow \infty$, for any $\lambda>0$, we can say that $|P(\xi)|^{\lambda} \hat{f}$ has some smoothness, and even some growth restrictions since

$$
\dot{B}_{p 1}^{n / p} \subseteq A_{p^{\prime}}, \quad 2<p,
$$

where

$$
A_{p}=\left\{f=\sum_{j=1}^{\infty} f_{j}^{*} g_{j} \mid \sum_{1}^{\infty}\left\|f_{j}\right\|_{p}\left\|g_{j}\right\|_{p^{\prime}}<+\infty\right\}, \quad 1<p<2
$$

and

$$
A_{2}=F L^{1} \subseteq A_{p}, \quad 1<p \leqslant 2 .
$$

We conclude with two remarks. First, it is sometimes possible to squeeze powers of $|x|$ in such a way that $|x|^{\rho} T^{\lambda} f \in L^{1}$.

TheOREM 4.9. Suppose $\lambda>(n-1) / 2,0<2 \rho<\lambda-(n-1) / 2$. Then

$$
\left(1+|x|^{2 \rho}\right) T^{\lambda} f(x) \in L^{1}\left(R^{n}\right) .
$$

The proof is similar to the proof of Corollary 2.7.

Our second remark concerns pseudodifferential operators. The symbol of a pseudodifferential operator is of class $S_{\rho, \delta}^{m}$ if there exist constants $C_{\alpha, \beta}$ such that

$$
\left|D_{\xi}^{\alpha} D_{x}^{\beta} p(x, \xi)\right|<C_{\alpha, \beta}(1+|\xi|)^{m-\rho|\alpha|+\delta|\beta|}, \quad \xi \in R^{n}
$$


where $0 \leqslant \delta<\rho \leqslant 1$. The associated pseudodifferential operator is

$$
p(x, D) f(x)=(2 \pi)^{-n} \int p(x, \xi) e^{i\langle x, \xi\rangle} \hat{f}(\xi) d \xi .
$$

TheOREM 4.10. If $f \in C_{c}^{l}, p \in S_{\rho, \delta}^{m}, l>m+n$, then

$$
p(x, D) f \in L^{1}\left(R_{n}\right) \text {. }
$$

Proof. It is enough to prove that

$$
\left(1+|x|^{n+\varepsilon}\right) p(x, D) f(x) \in L^{\infty}\left(R_{n}\right) .
$$

In fact, if $2 r$ is the smallest even integer larger than $n,\left(1+|x|^{2 r}\right) p(x, D) f(x)$ is bounded. Observe first that the integral defining $p f$ is absolutely convergent if $l>m+n$. This follows because if $f \in C_{c}^{l}$,

$$
|\hat{f}(\xi)| \leqslant C_{l}(1+|\xi|)^{-l}
$$

and thus

$$
|p(x, \xi) \hat{f}(\xi)| \leqslant C(1+|\xi|)^{m-l},
$$

and the right-hand side is integrable if $l>m+n$.

We also calculate

$$
|x|^{2 r} p(x, D) f(x)=(2 \pi)^{-n} \int\left(-\Delta_{\xi}\right)^{r}\left(e^{+i\langle x, \xi\rangle}\right) p(x, \xi) \hat{f}(\xi) d \xi,
$$

and we apply Green's formula repeatedly over a large ball, noting that $\hat{f} \in C^{\infty}$ and

$$
\left|D_{\xi}^{\alpha} \hat{f}(\xi)\right| \leqslant C(1+|\xi|)^{-l} \text {. }
$$

The boundary terms are finite sums of terms like

$$
D_{\xi}^{\beta_{1}} p(x, \xi) D_{\xi}^{\beta_{2} \hat{f}(\xi)}
$$

where $\beta_{1}+\beta_{2}=2 r$. The prior remark and the definition of $S_{\rho, \delta}^{m}$ show that the term is like $(1+|\xi|)^{m-\rho\left|\beta_{1}\right|-l}$, so that for $l>m+n$, this will also contribute a term tending to zero. This justifies the formula

$$
|x|^{2 r} p(x, D) f(x)=(2 \pi)^{-n} \int e^{i\langle x, \xi\rangle}\left(-\Delta_{\xi}\right)^{r}(p(x, \xi) \hat{f}(\xi)) d \xi,
$$

but again this is a finite sum of terms which are each $O\left(|\xi|^{-n-e}\right)$ at infinity and consequently

$$
\left(-\Delta_{\xi}\right)^{r}(p(x, \xi) \hat{f}(\xi)) \in L^{1}\left(R_{n}\right),
$$

which proves that $|x|^{2 r} p(x, D) f(x) \in L^{\infty}\left(R_{n}\right)$.

Taking $r$ larger, we see that we can also show, by assuming more differentiability on $f$, that $|x|^{2 r} D^{\alpha} p(x, D) f \in L^{1}$.

\section{REFERENCES}

1. T. Flett, On a theorem of Pitt, J. London Math. Soc. (2) 7 (1973), 376-384.

2. C. Herz, Lipschitz spaces and Bernstein's theorem on absolutely convergent Fourier transforms, J. Math. Mech. 18 (1968), 283-324. 
3. F. Keene, R. Lipsman and J. Wolf, The Plancherel formula for parabolic subgroups, Israel J. Math. 28 (1977), 68-90.

4. R. Lipsman and J. Wolf, The Plancherel formula for parabolic subgroups of the classical groups, J. d'Analyse Math. 34 (1978), 120-161.

5. B. Muckenhoupt and R. Wheeden, Weighted norm inequalities for fractional integrals, Trans. Amer. Math. Soc. 192 (1974), $261-274$.

6. H. Triebel, Interpolation theory, function spaces, differential operators, North-Holland, Amsterdam, 1978.

Department of Mathematics, University of Maryland, College Park, Maryland 20742 